La relectura de la historia como instrumento de construcción de la identidad:

los jesuitas y Rosas

Francisco Javier Gómez Díez

páginas / año $12-n^{\circ} 29$ Mayo-Agosto / ISSN 1851-992X/ 2020

http://revistapaginas.unr.edu.ar/index.php/RevPaginas

\title{
La relectura de la historia como instrumento de construcción de la identidad: los jesuitas y Rosas
}

\section{The rereading of history as an instrument of identity construction: the Jesuits and Rosas}

\author{
Francisco Javier Gómez Díez \\ Universidad Francisco de Vitoria (Madrid) \\ j.gomez.prof@ufv.es \\ https://orcid.org/0000-0002-5948-6186
}

\section{Resumen}

Si desde su origen la Compañía de Jesús recurrió a la narración de su propia historia como instrumento para configurar su identidad, la tarea resultó de especial importancia en el siglo XIX, al enfrentarse a tres dificultades nuevas: interpretar la supresión total de la orden, decretada en 1773 por Clemente XIV; responder al orden político nacido de la revolución burguesa y entrar en relación con novedosos ideales culturales y científicos.

$\mathrm{Si}$ desde el primer momento muchos jesuitas, por propia iniciativa, narran sus viajes, experiencias y misiones, a medida que avanza el siglo XIX este esfuerzo se institucionaliza y se dota de una pretensión científica que busca también ser reconocida fuera de los círculos de la Compañía.

El artículo se aproxima a esta problemática analizando la relectura de la misión de Buenos Aires durante la presidencia de Juan Manuel de Rosas; relectura que, a partir de la crónica de Mariano Berdugo (1841), realizan los jesuitas José J. Cotanilla, Rafael Pérez, Pablo Hernández, Juan Isérn, Joaquín Gracia y Guillermo Furlong. Analizo las razones que impulsan la redacción histórica y, desde aquí, busco establecer la inserción identitaria de la Compañía restaurada.

Palabras clave

Compañía de Jesús; Historiografía; Memoria histórica; Juan Manuel de Rosas; catolicismo argentino

\footnotetext{
Abstract

The company of Jesus resorted to the narration of its own history as an instrument to configure its identity, the task was of special importance in the XIX century, when responding to three difficulties: How to assume the total suppression of the order, decreed in 1773 by Clement XIV ?; How to relate to culture, science and, specifically, history? How to solve the conflict with the political order born of the bourgeois revolution?

From the first moment we find Jesuits who, on their own initiative, narrate their trips, experiences and missions. As the nineteenth century advances, this effort is institutionalized and endowed with a scientific claim that also seeks to be recognized outside of the Company's circles.
}

Esta obra está sujeta a la Licencia Reconocimiento-NoComercial-CompartirIgual 4.0 Internacional de Creative Commons. http://creativecommons.org/licenses/by-nc-sa/4.0/ 


\section{Francisco Javier Gómez Díez}

The article aims to approach this problem by analyzing the rereading of the mission of Buenos Aires during the presidency of Juan Manuel de Rosas. Knowing the chronicle of Mariano Berdugo (1841), six Jesuits write again on the same subject: José J. Cotanilla, Rafael Pérez, Pablo Hernández, Juan Isérn, Joaquín Gracia and Guillermo Furlong. It analyze the reasons that drive the historical writing and, from here, seeks to establish the identity insertion of the restored Company.

Keywords

Society of Jesus; Historiography; IHistorical Memory; Juan

Manuel

de

Rosas; Argentine Catholicism

\section{Introducción}

La narración de la propia historia ha jugado un papel decisivo en la configuración de la identidad jesuita; una configuración dinámica que, con sus elementos de continuidad, se ha prolongado hasta la actualidad. Esta tarea resulta de especial importancia a la Compañía decimonónica que, restablecida en 1814, se enfrenta a múltiples desafíos. Su forma de hacer historia $-\mathrm{y}$ sus motivos - refleja su autopercepción y su relación con la sociedad. Si desde el primer momento encontramos jesuitas que, por propia iniciativa o a petición de sus hermanos, narran sus viajes, experiencias y misiones, a medida que avanza el siglo este esfuerzo se institucionaliza, retomando una ya larga tradición (Betrán 2010). La Compañía, con sus súbditos dispersos por el mundo y dedicados a muy diversas actividades, buscó con ahínco la integración de éstos. Las cartas edificantes (Palomo 2005) y el encargo, ordenado explícitamente por los superiores, de redactar historias de las distintas misiones (Alcántara 2010) fueron los instrumentos para lograrlo. Finalizando el siglo XIX, sin cambiar de objetivos, el esfuerzo se dota de una pretensión científica que busca generar resultados provechosos y comunicables a sujetos y realidades ajenos a la Compañía.

Al mismo tiempo, mientras elabora una imagen de unidad y solidez interna, la Compañía debe insertarse en una nueva realidad política, burguesa, liberal y nacionalista, y, muy especialmente, debe explicarse la supresión decretada en 1773 por Clemente XIV. Ésta, por cuestionar la misma identidad jesuita, es difícil de asimilar. Muchos jesuitas escriben abiertamente, en crónicas, diarios, historias o correspondencia, sobre diversas expulsiones, pero nada dicen de la supresión, le niegan cualquier validez o, en la mayoría de los casos, reducen al mínimo esta ruptura.

Pretendo aproximarme a esta problemática analizando la relectura de la misión de Buenos Aires durante la gobernación de Juan Manuel de Rosas; relectura que, a partir de la crónica escrita por su superior, Mariano Berdugo (1803, Sevilla - 1857, Roma), realizan seis historiadores jesuitas. Considerando lo que conscientemente cuentan y lo que callan, analizo las razones que impulsan la redacción histórica y, desde aquí, busco establecer la inserción identitaria de la Compañía restaurada. Se 


\section{La relectura de la historia como instrumento de construcción de la identidad: los jesuitas y Rosas}

trata de lo que cuentan y callan conscientemente; aquello que conocen y prefieren esconder y no lo que ignoran por estar fuera de su capacidad de comprensión o de las fuentes que puedan utilizar.

\section{Mariano Berdugo y su Historia secreta}

En 1814 -décadas después de haber sido expulsada de sus dominios por los monarcas católicos más poderosos y disuelta por Clemente XIV— la Compañía de Jesús fue restaurada por Pío VII, culminando un proceso, eclesiástico y político, iniciado tiempo atrás. A la sombra de la Restauración europea, la Compañía renace marcada por un fuerte talante antiliberal, con la simpatía de unos poderes deseosos de hacer frente a la revolución y con el rechazo de los principales partidarios de ésta. Así, en 1815 la restablece en España Fernando VII; es suprimida por las Cortes liberales en 1820; autorizada tres años después y, en 1835, tras sufrir, con otros religiosos, las matanzas de 1834, nuevamente disuelta, acusada de complicidad con el carlismo. En estas circunstancias, aunque el prepósito general, Philippe Roothaam, alentaba las misiones de Ultramar, los jesuitas españoles se dirigieron a América - primero a Buenos Aires y, poco después, a Nueva Granada- más forzados por la necesidad que deseosos.

Conociendo estos hechos, Juan Manuel de Rosas permitió que se les llamara a Buenos Aires. En agosto de 1835 llega un primer grupo. El apoyo episcopal y gubernamental - por muy interesado que fuese - llena de optimismo a Mariano Berdugo, que sueña establecer, con dicho apoyo y misioneros europeos, una provincia en torno a un colegio en Buenos Aires. La situación parecía prometedora. En febrero de 1837 inauguraron el colegio. Pese a las dificultades, no dejó de crecer: en 1840, un año antes de su clausura, tenía 85 alumnos internos y 250 externos ("La Compañía en Buenos Aires 1836-1843"; Archivo Histórico de Loyola; AHL. Fondo Lesmes Frías C. 33 n.ํㅜㄹ).

Los objetivos de Rosas y de los jesuitas terminarían siendo incompatibles. Rosas defendía una Iglesia de Estado - significativamente nunca derogó la reforma eclesiástica de Rivadavia- (Di Stefano 2006) y, tras comprender que aquellos no se plegarían a sus pretensiones, los expulsó en 1841. Refugiado en Montevideo, y quizás recurriendo al diario que, según Pérez (1901, p. 637n), acostumbraba a escribir, redacta Berdugo Historia secreta de la supresión de la Compañía de Jesús en Buenos Aires (1843) ${ }^{1}$. Recurriendo sólo a documentos o testimonios fidedignos, pretende ayudar — cuando otros emprendan esta tarea- a la redacción imparcial de la historia, responder a las calumnias vertidas contra los jesuitas y, evidenciando las tensiones que habían existido, explicar a sus súbditos las decisiones que había tomado. Al margen de lo que estos objetivos puedan hacer pensar, es un texto destinado a sus superiores.

\footnotetext{
1 Archivum Romanum Societatis lesu (ARSI) Arg-Ch 1001, II, 9. En su momento, cito los parágrafos numerados por Berdugo. Preparo una edición crítica de este texto, un manuscrito de 102 páginas.
} 


\section{Francisco Javier Gómez Díez}

Comienza con una anécdota. Nada más desembarcar en Buenos Aires se forzó al grupo de jesuitas a colocar sobre sus ropas un distintivo político rosista. Berdugo dice haber temido, desde ese momento, que la comunidad jesuita fuese condicionada por intereses partidistas. Sus primeras cartas desde Buenos Aires, y las que escriben sus compañeros, no recogen esta anécdota y sí manifiestan lo bien que fueron recibidos (Cfr. AHL fondo Lesmes frías caja 025, n. 118). Quizás anticipa temores surgidos posteriormente para presentar, desde el principio, el núcleo de su obra: la pretensión de Rosas de subordinar la Compañía a su política, la lucha de ésta por conservar la independencia y, en consecuencia, el inevitable conflicto. Mientras, los jesuitas desarrollan sus ministerios con éxito y apoyo popular, el colegio prospera y el pueblo, federal y unitario, confía en ellos, en gran parte porque siendo extranjeros - algo positivo- son ajenos a las contiendas políticas, siempre connotadas negativamente. Insiste Berdugo, orgulloso, en su españolidad y, reconociendo otra rivalidad, critica al clero local sus costumbres y su subordinación a Rosas. Dudando, en ocasiones, de la sincera religiosidad del Gobernador, le repudia su pretensión de instrumentalizar la religión, contando con la mayoría del clero y, muy especialmente, con el apoyo de monseñor Medrano, al que, por su avanzada edad y ceguera, describe Berdugo, bienintencionado, pero condescendiente, débil y manejable, es decir, "una verdadera nulidad" [§ 39].

Con un pretendido apoliticismo señala, varias veces, que no pretende juzgar la política de Rosas; solo quiere exponer aquello que contribuya a explicar la situación de los jesuitas. Aun así, incorpora observaciones sobre el sistema imperante y los modos de actuación del gobierno, cuyo violento proceder se extrema, día a día, al tiempo que crecen las amenazas contra su estabilidad. Identificada plenamente la Federación con su dirigente, Berdugo considera que el régimen es despótico, recurre permanentemente al espionaje, a las amenazas y a la violencia y, en ocasiones, al asesinato político. Destaca el papel de tres instituciones (la Policía, la Sociedad Popular Restauradora o mazorcay el Cuerpo de Serenos) que, con terrible violencia, amedrentan al pueblo. En definitiva, Rosas "por conseguir lo que se propone no repara en medios" [§ 22].

Rosas multiplica sus injerencias en el gobierno jesuita -ordena predicar la sumisión a la Federación, envía a las misiones populares divisas federales para repartir entre los fieles, se preocupa por la indumentaria de los alumnos del colegio, etc.- y Berdugo se resiste: busca reducir esa predicación a una sincera aceptación del orden establecido; se niega a repartir las divisas en las misiones; reprende a uno de sus súbditos, el padre Francisco Majesté, por excederse en la defensa del federalismo... Teme, en resumen, que la más mínima cesión dé pie a mayores y mayores exigencias.

La situación se va tensando y, ante la resistencia jesuita, Rosas modifica su estrategia. Si al principio esperó, casi vio natural, la subordinación jesuita, poco a poco fue manifestando, en quejas cada vez más duras, su frustración: lamenta que los jesuitas no le visiten con más frecuencia; interrumpe las misiones populares cuando el padre Gandásegui — según Berdugo por un despiste- no predica la 


\section{La relectura de la historia como instrumento de construcción de la identidad: los jesuitas y Rosas}

sumisión a la Federación. Sus extraoficiales emisarios empiezan a comunicar a los jesuitas ese malestar: la mujer de su hermano, Prudencio, que se confesaba con el padre Cabeza, le refiere lo enojada que estaba la mazorca y el riesgo que, por lo mismo, corrían los jesuitas; José Rafael Reina — sacerdote amigo de los jesuitas, negociador de su llegada y, al tiempo, muy próximo a Rosas (ARSI, ARG-CHIL, 1001III,1) - y el obispo auxiliar, monseñor Escalada, les piden que reconsideren su actuación, argumentando con los daños que acarrearía su marcha.

Rosas confía en rendir por miedo a los jesuitas, que, ya en julio de 1839, han caído en desgracia $\mathrm{y}$, abiertamente, comienzan a ser criticados, primero, y amenazados, después. En una de las audiencias que les concede pide al padre Parés que tengan cuidado con su proceder o, quizás, él no podría contener a la mazorca; todos entendieron la amenaza.

Tras frustrar los proyectos que Berdugo le expuso para distribuir a los jesuitas por toda la república - prohíbe las misiones populares, pospone sine die la evangelización de los indígenas e ignora las solicitudes de otras provincias-, Rosas inicia una nueva estrategia: dividir a la comunidad jesuita, divulgando que los padres Majesté, García y Cabeza son federales, mientras Berdugo y los demás son unitarios.

Las amenazas crecen y, con ellas, el miedo de los jesuitas. Mientras la mazorca moviliza todos sus recursos, Rosas plantea un ultimátum. Así interpreta Berdugo la conversación que mantuvo con Pedro de Angelis, el periodista napolitano que era, ya por entonces, uno de los principales propagandistas de Rosas (Lozier 2018). Le hace saber que sería una lástima perder todo lo logrado y arriesgar su seguridad personal cuando todo podía resolverse nombrando superior a Majesté, más acorde con los deseos de Rosas. La propuesta era inviable y la ruptura, inevitable.

El 5 de octubre Berdugo expuso a la comunidad la situación. Ésta, atemorizada, se niega a permanecer reunida esperando un inminente golpe. Berdugo autoriza a los suyos a buscar refugio en casas particulares. Él lo hace en la de monseñor Medrano, donde descubre la conspiración que Rosas ha preparado, con el apoyo de la jerarquía eclesial y la complicidad de Majesté, para reemplazarle — sin contar con los superiores romanos - al frente de los jesuitas. Al día siguiente, con los padres dispersos y el colegio vacío, Berdugo escribe por última vez al Gobierno; denunciando indefensión, le devuelve la iglesia y el colegio. Abandona la comunidad, da instrucciones a los suyos y huye a Montevideo. Cerrado el colegio y huido Berdugo, la mazorca, como cumpliendo órdenes, no repite más sus amenazas.

La grave situación no impide a Berdugo reconocer los límites de Rosas: afirmándose protector de la religión, no podía actuar directamente contra los jesuitas; sólo cabía temer las imprevistas consecuencias de una violencia descontrolada.

Sin Berdugo, Rosas orquesta dos maniobras para reagrupar y subordinar a los jesuitas. Planea, en primer lugar, reabrir el colegio (con los mismos maestros y otra dirección). Sus agentes - ministros y eclesiásticos - comienzan a tantear el terreno y Majeste, ya abiertamente rosista, hace una propuesta a los padres Coris, Sató y Gomila. La salida de Buenos Aires de los dos primeros desbarató el plan. La segunda 


\section{Francisco Javier Gómez Díez}

maniobra consistió en abrir, a principios de febrero, la iglesia bajo la rectoría de los padres Majesté y Cabeza. Aunque los jesuitas accedieron, se puso de manifiesto la grave división que les afectaba.

Berdugo, desde Montevideo, busca una solución definitiva: mantiene, en la medida de lo posible, comunicación con sus súbditos; va retirando, en la forma y ritmo que puede, a algunos sujetos de Buenos Aires y expulsa de la Compañía a los padres identificados con Rosas: Ildefonso García y Francisco Majesté. Entonces, la Providencia, dice Berdugo, resuelve el conflicto. Habiendo Majesté abandonado la Compañía, Cabeza solicita a Rosas que le exima de toda responsabilidad en la iglesia de San Ignacio. Rosas - quizás convencido de que nada le queda por hacer-impone a los jesuitas la secularización o el abandono de Buenos Aires. Sólo tres se quedan: Majesté, García y Baylón.

Si las presiones de Rosas y la resistencia jesuita son uno de los ejes de la Historia, el otro, más importante para Berdugo, es el efecto que esto pudo tener en la unidad y disciplina de su comunidad.

«Es de advertir, escribe, que he tenido el sentimiento de ver en los míos, que mis anuncios, y aun disposiciones no eran creídas, ni recibidas con la debida deferencia de juicio. Sea que me consideraban de pocos alcances, o prevenidos de prejuicios, sea, que teniendo el concepto algo ventajoso de los suyos, y de su disposición para no venir en él; sea, que el interés de los dos disidentes echaba un velo, para no verlo; lo cierto es que no había persona más examinada que la del superior, ni más criticada en sus disposiciones, según que cada uno veía las cosas, o quería, que el superior gobernase. De donde entre los nuestros se hablaba, discurría, condenaba, aprobaba a cerca de todo, hasta entre los hermanos coadjutores, que no faltó, quien pretendiese también las borlas. Y de aquí no dejó de rezumar algo, o algos hasta los seglares, con quienes conversaban y vivían». [§ 55]

Esta cuestión no está presente en las historias posteriores. Reducen al mínimo las tensiones internas que afectaron a la comunidad jesuita, elogiando la obediencia, la unidad y el sacrificio de los sujetos, o de la inmensa mayoría de ellos. Si Berdugo constata la división de la comunidad, los textos posteriores luchan contra ella escondiéndola.

Berdugo reconoce la existencia de sujetos ejemplares que supieron edificar a la comunidad. Los hubo también abiertamente rebeldes y otros, los más, problemáticos, díscolos o inestables. Entre los rebeldes, Ildefonso García merece las críticas más graves: intimó en exceso con algunos clérigos; se declaró en abierta desobediencia y, al parecer, se unió sin recato con una mujer. El caso de Francisco Majesté es más complejo. Entresacando las numerosas opiniones que sobre él vierte, puede afirmarse que Berdugo le considera, fundamentalmente, vanidoso y de insensata inocencia. Por esta razón fue fácilmente seducido por los rosistas, que le prodigaron elogios y alabanzas, consiguiendo, primero, que defendiera hacer concesiones y, luego, que cayera en el disimulo con su superior, en la defensa de ideas mundanas y en la desobediencia. 


\section{La relectura de la historia como instrumento de construcción de la identidad: los jesuitas y Rosas}

Berdugo no escatima críticas contra otros jesuitas. Denuncia que, en los meses de dispersión, si se condujeron bien a los ojos de la población, estuvieron muy lejos de satisfacerle a él que los conocía mejor. De este modo, concluye — pesimistacuestionando el fundamento mismo de la misión. Considera mejor abandonarla que, como parece que se ha hecho, poner en riesgo el crédito de la Compañía enviando jóvenes sin letras ni talento $\mathrm{y}$, sobre todo, sujetos de escasa "obediencia, mortificación y espíritu". [§ 85]

La franqueza con la que se expresa es proverbial. Su obra no es una historia pensada para su publicación. Es un informe a los superiores para explicar una política y, quizás, ayudar al futuro gobierno. Es necesario señalar que todos aquellos que reescriben esta historia lo hacen con la intención de ver su trabajo publicado.

\section{Los historiadores del conflicto}

Los autores que recrean la misión bonaerense se integran en una continuidad historiográfica que, pasando por el gran número de diarios, crónicas e historias redactadas por los misioneros decimonónicos, va de la relectura del hecho americano por los jesuitas expulsos en el último tercio del siglo XVIII (Batllori 1966; Guasti 2006), a la construcción de una historiografía de perfil nacionalista a mediados del siglo XX (Cárdenas \& Di Stefano 2014). Al comienzo los jesuitas escriben por propia iniciativa y para ellos; lentamente lo van haciendo por encargo de los superiores y, no sabemos con qué éxito, también para sectores ajenos a la Compañía.

Seis son los autores que me han interesado.

José J. Cotanilla (Toledo, 1818 - Madrid, 1886) escribe, por propia iniciativa, una obra inédita: Historia de la misión colombiana de la Compañía de Jesús (1866), donde dedica cuatro capítulos a la misión bonaerense $\left(12^{\circ}, 13^{\circ}\right.$, $14^{\circ}$ y $\left.16^{\circ}\right)$. Conozco tres copias manuscritas, dos en el Archivo de la Provincia de España de la Compañía de Jesús de Alcalá de Henares (AESI-A C-92 y C-96) y otra en el Archivo Histórico de Loyola (AHL Misiones Caja 9, núm. 1) (sobre estos manuscritos, Gómez Díez 2016). Cotanilla es un misionero de ideología integrista y ferozmente antiliberal. Ignoro, pero no descarto, que esto tenga algo que ver con que no se haya publicado su obra, llena de información muy aprovechada, posteriormente, por Rafael Pérez. Berdugo fue su maestro de novicios y, como él, sufrió la trágica experiencia de las masacres de Madrid (1834) y diversas expulsiones. Cotanilla no leyó la Historia secreta y es muy probable que ignorara su existencia, pero, como demuestra la lectura de su obra, tuvo buena información sobre los sucesos de Buenos Aires. Se la proporcionaron sus 20 años en América, el contacto y amistad con muchos misioneros y, muy especialmente, su relación con el $\mathrm{H}$. Saracco, uno de los sujetos que más cerca estuvo de Berdugo durante los primeros años cuarenta.

Muy distintos son Rafel Pérez (Guatemala, 1842 - Barcelona, 1901) y Pablo Hernández (Teruel, 1852 - Roma, 1921). Al margen de la temprana expulsión de Centroamérica del primero, sus vidas fueron más tranquilas que las de Berdugo y 


\section{Francisco Javier Gómez Díez}

Cotanilla. Escribieron sus obras por encargo de sus superiores. El primero conoce la Historia secreta; la cita y extracta con profusión. Hernández, como Isérn, Gracia y Furlong, conozca o no conozca la obra de Berdugo, se apoya fundamentalmente en la de Pérez, un voluminoso texto de casi mil páginas. A la misión bonaerense dedica toda la primera parte, 293 páginas, $y$, posteriormente, va rastreando las actividades del P. Berdugo hasta su fallecimiento (segundo capítulo de la tercera parte, donde incorpora un elogio conclusivo sobre él). La obra de Hernández (1914) es en gran medida una síntesis de la de Pérez: unas 300 páginas; menos de 30 dedicadas a la misión bonaerense y, al final del libro, una nota biográfica sobre Berdugo.

Juan Isérn (Barcelona, 1870 - Buenos Aires, 1941) y Joaquín Gracia, también español y un año más joven, sin hacer de esta actividad el eje de su apostolado, prolongan el hacer historiográfico de Pérez y Hernández con dos aportaciones específicas, en las que el gobierno de Rosas es una cuestión tangencial: algo más de treinta páginas (el capítulo tercero) en la de Isérn (1936), para un libro de más de quinientas, y unas 80 páginas en el caso de Gracia (1940), teniendo su obra setecientas cincuenta. Por último, Guillermo Furlong (Santa Fe, 1889 - Buenos Aires, 1984), uno de los historiadores más prolíficos de toda la Compañía de Jesús, se aproxima - también de forma tangencial - en repetidas ocasiones a Rosas y, sin duda, conoce el texto de Berdugo. Con independencia de su lugar de nacimiento, no cabe ignorar que estos tres últimos autores se encuentran fuertemente integrados en la realidad nacional argentina y no se sienten, como los anteriores, meros misioneros coyunturalmente establecidos en Argentina.

Antes de analizar lo que escriben sobre la misión bonaerense, es conveniente determinar el marco político y eclesiástico en el que la insertan, así como su concepción de la historia y su método.

Como toda la historiografía jesuítica, estos autores desarrollan una lectura providencialista de la historia, donde la Compañía es un instrumento esencial al servicio de la comunidad eclesial. "Dios sabe sacar bienes —escribe Cotanilla- y grandes bienes de los mismos medios de que se valen los hombres perversos, y con intención perversa de sacar males y grandes males". Así, las diversas supresiones y expulsiones, incluida la de Buenos Aires, y el sufrimiento de unos cuantos, como el de los primeros mártires, contribuyen a la difusión del Evangelio. La Compañía, víctima de los golpes más duros, es avanzada sufriente de la Iglesia en lucha contra "los hijos de la impiedad". El mismo planteamiento desarrolla Rafael Pérez, aunque buena parte de los durísimos textos antiliberales e integristas presentes en la obra de Cotanilla han desaparecido en la de un guatemalteco veinticuatro años más joven, que escribe ya durante el pontificado de León XIII.

Isérn (1936, p. 100-2) presenta una versión menos apasionada. Vincula, como sus predecesores, los sucesos españoles de 1834 y 1835 con la llegada de los jesuitas a América, pero, al tiempo, amplía la perspectiva. Observa la coincidencia temporal de la transformación política de Europa, las persecuciones sufridas por las órdenes religiosas y la extensa penetración colonial europea que abre a éstas nuevos campos de acción misionera. 


\section{La relectura de la historia como instrumento de construcción de la identidad: los jesuitas y Rosas}

Cotanilla prácticamente niega que la Compañía fuese suprimida (Gómez Díez 2018), como también hará Gracia, que no concede valor a la bula de Clemente XIV por haber sido firmada a la fuerza y señala, insistentemente, que la Compañía se prolongó, viva, en Rusia y con legitimación papal, hasta el decreto de Pío VI. Pérez, Hernández e Isérn simplemente la silencian, limitando sus comentarios a la expulsión de 1767. Furlong, reconociéndola, la diluirá rápidamente en la supervivencia de la Compañía en Rusia, con el beneplácito de Pío VII. Actúen de una u otra forma, todos insisten en la continuidad entre la antigua Compañía y la nueva, invocando a los ancianos que, vivos, vinculan a ambas o al recuerdo agradecido de un pueblo que recibió la bendición de la antigua y añora el retorno de la moderna. A este respecto es básica la función que cumplen las reducciones paraguayas (Telesca \& Perrone 2014; Gómez Díez 2016) Explícitamente Isérn presenta a Mariano Berdugo como el garante de la continuidad:

"Él fue el primer novicio del noviciado abierto en Sevilla, del cual era rector y maestro de novicios el P. Gaspar de la Carrera, anciano de 76 años de edad, perteneciente a la antigua Compañía. En la apertura del noviciado, en que vistió la sotana el P. Berdugo presidió el acto el P. Manuel de Zuñiga, otro anciano de 70 años de edad, quien había asistido en Roma, a la lectura de la bula de Pío VII, restableciendo la Compañía el 7 de agosto de 1814 y era entonces comisario general de España. Estos hechos demuestran la vinculación del P. Berdugo primer superior de los nuevos jesuitas en la Argentina, con los padres de la antigua Compañía; lo cual es una especial garantía de la legítima sucesión espiritual entre los antiguos y los nuevos jesuitas". (Isérn 1936, p. 108)

La transformación del discurso en torno al proceso de independencia americana es también significativa.

Cotanilla, que, no obstante, sólo escribe para jesuitas, en su mayoría españoles, para sus simpatizantes y bienhechores, ve en la independencia una tragedia social. Pérez defenderá lo mismo: la emancipación, originada en principios que habían sembrado de ruina Europa, dio origen a la anarquía y a la disolución social; ven en ella un trágico proceso revolucionario que, por influjo ilustrado y francés, traicionó la identidad católica americana. Solo autores posteriores y no españoles modificaran estas tesis. Gerard Decorme -francés firmemente arraigado en la provincia mexicana- vincula la expulsión de los jesuitas con la independencia americana por causa del agravio sentido por los criollos y, años después, Furlong considera a los jesuitas responsables activos de la "emancipación". Esta transformación no es ajena ni a la consolidación de las repúblicas americanas ni al origen nacional de los jesuitas que en ellas trabajan.

Todos, sin renunciar nunca a una pretensión moralizante, van definiendo una concepción de la historia centrada en la reconstrucción de la verdad a partir de documentos cuyo valor reside, en gran parte, en la autoridad de sus redactores. Cotanilla - aunque reconoce que ha censurado documentos - habla de una verdad documentada sobre textos manuscritos e impresos que le han proporcionado, principalmente, sus propios hermanos. Pérez, desarrollando esta idea, progresa en 


\section{Francisco Javier Gómez Díez}

la definición de su condición científica. Su obra nace de un encargo explícito de sus superiores y ha contado con la colaboración de múltiples jesuitas y con su presencia en el teatro mismo de los hechos. Está en marcha una empresa historiográfica internacional y colectiva; historiográfica y archivística. La empresa que impulsa el P. General Luis Martín y da sus frutos en el Instituto Histórico Societatis Iesu (Morales 2014).

Pérez, en el prólogo, especifica las fuentes de su investigación: lo poco que se ha escrito sobre los jesuitas, las obras de historia general que tienen algún punto de contacto con su asunto $y$, principalmente, fuentes primarias: documentos oficiales $y$ manuscritos o testimonios orales de personas autorizadas. Además, ha recopilado, conservado, clasificado y numerado estos documentos "para poder citarlos debidamente, no sea más que con el título de colección particular". Reconoce que sólo cita los documentos cuando reproduce palabras textuales, para no multiplicar las citas como las líneas de su escrito porque dice no tener más conocimiento de los hechos que el que proporciona el estudio de los documentos. Además, concluye, la veracidad de una obra no la garantiza el número de las citas, "que nadie se empeñará en verificar", sino la buena fe, el buen criterio y la esmerada diligencia del escritor. Esto debe abalar su obra y la autoridad de sus fuentes, como demuestra al fundamentar el valor documental de la Historia secreta en "las virtudes nada vulgares" de su autor, el puesto de superior que ocupo y su exactitud y constancia en anotar todos los sucesos que ocurrían. En definitiva, "podría acaso equivocarse en sus juicios, pero no en la fiel exposición de los acontecimientos, que es lo que más hace a nuestro propósito". Nos encontramos, de forma evidente, ante la versión jesuita confesional de la escuela metódica desarrollada, desde el último cuarto del siglo XIX, por Langlois, Seignobos y Ranke.

Con respecto a los documentos, la misma posición adoptarán los demás autores. Mientras identifican la verdad histórica con la exacta reproducción documental, van encadenando los mismos textos, citándolos expresamente o no. Isérn, también en el prólogo, lo justifica: "Con esta abundancia de materiales difícilmente puede extraviarse la narración; más aún, con facilidad se puede convertir ella misma en un tejido continuado de textos originales, como sucede en algunos capítulos de esta obra; razón por la cual se omite frecuentemente la cita, que podría añadirse a cada frase".

Como argumenta Perla Chinchilla (2014) esta pretendida objetividad científica no anula los objetivos edificantes y propagandísticos, los refuerza con el apoyo de la "verdad documental", pero, en mi opinión, sirve, además, como recurso retórico de identificación con la comunidad científica laica. Es significativo que, paralelamente se desarrolle un esfuerzo por ampliar los destinatarios de sus obras. Si Cotanilla reconoce que ha alterado los documentos para eliminar todo aquello que pueda resultar injurioso incluso al desgraciado que cometió hechos execrables y tiene conciencia de no estar escribiendo para un público amplio, los demás —en lo referente a documentos y lectores- dicen pretender lo contrario. Hernández, en el seno de una renovación historiográfica general, dice escribir tanto para los de 


\section{La relectura de la historia como instrumento de construcción de la identidad: los jesuitas y Rosas}

dentro como para los de fuera, tanto para jesuitas, que, estimulados por el ejemplo de sus predecesores, busquen imitarles, como para futuros historiadores y público en general. Esa será la posición de los posteriores, pues nadie, creyente o no, piensan- podría negar la inmensa contribución de la Iglesia a la civilización de los pueblos y, en el caso concreto de Furlong (1933, p. 176), a la independencia de los pueblos americanos, que califica de causa nacional a la que era justo subordinar otras urgencias.

\section{La historiografía jesuita frente a la misión bonaerense}

Si la misión bonaerense se desarrolló en un periodo de inestabilidad, la situación había cambiado considerablemente cuando empezaron a publicarse las crónicas jesuitas sobre la misma. Mientras, por primera vez, empieza a cuestionarse la imagen que de Rosas había construido la historiografía liberal, en torno a 1880 concluiría para la iglesia una época marcada por la inseguridad. Se inicia un periodo de desarrollo, reformas institucionales y renovación del pensamiento católico. Entre 1890 y 1920 se recomponen las relaciones entre la iglesia y el estado, facilitado por el clima ideológico de fin de siglo y el temor de las élites a los problemas asociados a la conflictividad social y las masas de inmigrantes. El estado y la iglesia tomaron conciencia de la imposibilidad de extender, sin colaborar, su influencia sobre la sociedad (Imolesi 2014). Al tiempo, la historiografía busca desarrollarse como ciencia y, tanto la Iglesia como la Compañía de Jesús, ambicionan participar en este proceso (Morales, 2014).

Cotanilla, aprovechando que, tras su salida de Buenos Aires, encadena sucesivas responsabilidades como superior, convierte a Berdugo, como harán los autores posteriores, en el protagonista principal de la misión, en torno al que permanece unida el resto de la comunidad. Con el apoyo de la necrológica que se le dedica en Roma, reproducida íntegra por Cotanilla y, posteriormente, por Pérez, una serie de rasgos le caracterizan: su "virtud y letras", su prudencia y firmeza; su puntualidad, modestia y obediencia y, especialmente, el amor que hacía él sintieron todos sus súbditos. Su firmeza, el no haber querido condescender con las pretensiones de Rosas, "ni comprometer su sagrado ministerio", es el origen del conflicto. En los textos posteriores Berdugo conserva esta imagen, fundamento del elemento más estable de todo este proceso de relectura histórica: la firme unidad jesuita en torno al superior.

Rosas, por el contrario, es reinterpretado. Cotanilla critica su pretensión de obligar a los jesuitas a predicar en su beneficio y le considera un tirano ambicioso y sanguinario, pero, y esto es lo significativo, no establece ninguna distinción entre él y todos los enemigos decimonónicos de la Compañía: Morazán, Francia, Obando, Juárez, Urbina o Mosquera; todos son, sin más, liberales y es difícil encontrar en Cotanilla un adjetivo peor para calificar a sus enemigos. Basta citar el capítulo 17 de su Historia, donde no duda en escribir: "Un Lucifer, un Adán, una mujer engañada, un envidioso... casi se podría demostrar, en esos cuatro tipos primitivos del 


\section{Francisco Javier Gómez Díez}

liberalismo, el grado mayor o menor de la tan maldita secta, en que se encuentran enumerados los liberales de todos los tiempos".

$\mathrm{Si}$, tras el inicial apoyo gubernamental, comenzó Rosas a dar muestras de descontento porque no predicaban lo que él llamaba causa santa de la federación, el conflicto se habría podido evitar —escribe Cotanilla consciente de la tradición antijesuita de su tiempo- "si los jesuitas hubieran sido tan mundanamente políticos como lo suponen sus enemigos, adhiriéndose al partido del liberalismo Rosas". Así, en 1841, la situación es ya irreversible: comenzaron a salir de la casa del "tirano, cuadrillas de satélites llamados de la Mazorca, gritando por las calles y bajo las ventanas de nuestro mismo colegio" y, el 4 de octubre, Berdugo dispersó a su comunidad. Cotanilla calla que Berdugo había acusado a sus súbditos de forzarle a permitirles dispersarse y prolonga la narración ignorando cualquier tensión en el seno de la comunidad jesuita. Huido Berdugo los padres no fueron molestados en lo más mínimo y, mientras duró la dispersión, hasta marzo de 1843, Rosas hizo inútiles tentativas para que se reunieran otra vez en el colegio, aunque de un modo cismático. Fracasado su intento, los expulsó.

Las reflexiones del capítulo 16 prueban que Cotanilla estaba relativamente bien informado de lo sucedido. Explica las desgracias y dificultades a las que se enfrentaron los jesuitas, pero, sobre todo, insiste en lo que realmente les aflige: la ofensa a Dios, la pérdida de las almas y la ruina de algunos hermanos que perdieron su vocación "en esos días aciagos de trastornos y de revolución”, pese a los esfuerzos de los superiores, "cual padres solícitos, como madres bondadosas y tiernas", para conservarles en el seno de la Compañía. Termina reproduciendo dos cartas. En una, un expulso reconoce "lo que sin duda alguna debieron sentir también los demás", que el P. Berdugo, tenía toda la razón y ellos eran los engañados. La otra refuerza la misma idea. Berdugo, el 3 de enero de 1853, informa de la desgraciada suerte de aquellos que, una vez abandonada la Compañía, fueron perseguidos, criticados, ignorados e, incluso, llegaron a enloquecer. El sentido de este capítulo sólo se alcanza teniendo presente que la obra de Cotanilla está escrita, principalmente, para los jesuitas y, al tiempo, buena parte de los protagonistas siguen vivos y trabajando en América. Igualmente, cabe consultar la carta original (AHL Fondo Lesmes Frías, caja 34, n. - 28) para constatar que ésta carece del tono tremendista con que la tiñe Cotanilla.

El tono varía considerablemente en la obra de Pérez. Berdugo sigue siendo el protagonista, obediente, firme, unido a Roma y gozando de la plena confianza de ésta, pero Rosas no recibe una crítica tan falta de matices como la de Cotanilla. Pérez considera, en primer lugar, que unitarios y federales adoptaron una política terrorista y, si Rosas fue un tirano sanguinario, rechaza dar sobre él un juicio categórico; esta difícil cuestión "acaso con el tiempo tendrá una resolución definitiva", pero, por ahora, "solo encontramos en los escritores argentinos -a ninguno de los cuales cita- apreciaciones contradictorias" (Pérez, 1901, p. 34). Reconoce su origen familiar ilustre y rico, lo que, sin duda alguna, es motivo de alabanza; su aire gauchesco y sus no pocas virtudes: maneras finas y hasta 


\section{La relectura de la historia como instrumento de construcción de la identidad: los jesuitas y Rosas}

agraciadas, facilidad de la expresión, cultura en su trato e ingenio no vulgar en el manejo de los asuntos, no solo económicos, sino también políticos. Este esquema de virtudes es muy significativo por su cercanía a lo promovido en la formación decimonónica de los alumnos en sus colegios. Distingue entre el primer periodo de gobierno, que desempeña con acierto, apoyos y rasgos de celo religioso dignos de García Moreno o Carrera, y el segundo, que describe como terrorista, ya fuese como resultado de las circunstancias en que le colocaron sus enemigos o por un plan que tenía preconcebido. Aun así, reconoce que no golpeó con más violencia a los jesuitas porque necesitaba presentarse como defensor de la religión.

La fuente principal de Pérez es la Historia secreta, cuya autoridad se establece invocando las virtudes de su autor y la opinión favorable que de él tuvo la autoridad romana. Sigue y parafrasea esta Historia, reproduciendo los sucesos más graves y los aparentemente más insustanciales, pero poco queda de la tensión en el seno de la comunidad jesuita, ni de los fallos o la debilidad de sus miembros. En contrapartida, es muy crítico con el clero nacional: mediocre, enfrentado a la Compañía y comprometido plenamente con Rosas. Evidentemente se trata de una obra de consumo interno escrita en España.

Con Berdugo, el jesuita que más interés despierta en Pérez es Francisco Majesté. Ve en él a un hombre de talento y carácter festivo, gran orador y amigo de complacer, pero no hace esfuerzo alguno por explicar ni las razones de su proceder ni las causas de su expulsión. Busca dejar claro que sus superiores hicieron todo lo posible para conservarle en el seno de la orden porque la Compañía sólo recurre a medios extremos después de haber agotado todos los recursos ordinarios y extraordinarios. Con la intención de probar que el único responsable fue Majesté, reproduce las cartas que sobre el tema escribieron Roothaan y Berdugo. Su interés, frente al silencio con respecto a los otros sujetos que abandonaron la Compañía, debe relacionarse con su éxito posterior. Es muy significativo que Pérez $-\mathrm{y}$ sus continuadores - calle algo que debía saber: tanto Majesté como García terminaron reconstruyendo unas relaciones cordiales con la Compañía (Fernández Techera 2007, pp. 42 y 72), en el caso del primero desde la posición ventajosa que le proporcionó una carrera eclesial exitosa. Tras la caída de Rosas, en 1852 pasa a Montevideo, donde gozó, hasta su muerte, de una gran popularidad como predicador y fue fiscal eclesiástico y catedrático de derecho canónico con el apoyo de monseñor Jacinto Vera, discípulo, muy apreciado, de los padres jesuitas.

En definitiva, Pérez presenta un conflicto marcado por la llegada de los jesuitas a una tierra que les necesitaba; la imposibilidad de extenderse por la República porque a ello se opuso Rosas; la resistencia firme de la Compañía a las presiones del Gobernador y la firme unidad jesuita en torno a su superior, representante de la autoridad romana.

Hernández, que había vivido en Argentina y escrito, en repetidas ocasiones, dirigiéndose a sus ciudadanos - hay que recordar especialmente su Juicio crítico (Hernández, 1886) —, sigue fielmente la obra de Pérez; no cita, quizás no ha leído, la de Berdugo. Establece, de forma más sistemática, precisiones en torno al gobierno 


\section{Francisco Javier Gómez Díez}

de Rosas, distinguiendo entre él y el partido que representaba; entre éste, más tradicional por religioso y por asumir la herencia española, y el unitario, que pudiera llamarse francés; la forma en la que llevo adelante sus pretensiones y sus dos periodos de gobierno. Precisiones al margen, tacha el proceder de Rosas de tiránico, bárbaro, salvaje y contrario a la ley natural y la humanidad y, siendo así, era imposible que los jesuitas subsistieran en Buenos Aires. Elogia los ministerios desarrollados por éstos y el firme apoyo popular del que gozaron, como confesores, por el buen nombre que heredaron de los antiguos padres, su condición de extranjeros y su disponibilidad para atender a los penitentes; como predicadores, destacando en esta faceta el padre Majesté; como misioneros populares y, muy especialmente, como profesores. Siendo Berdugo nuevamente el protagonista de la historia - ejemplo de virtudes, de obediencia y fiel ejecutor de los deseos de Roma-, lo realmente interesante es como se reduce, prácticamente hasta desaparecer, la tensión en el interior de la comunidad jesuita. Veamos el contraste. En 1843, tras huir a Montevideo, había dejado Berdugo

"distribuidos a los nuestros de suerte que pudiesen ayudarse con consejos y ejemplos los que habitasen en casas más cercanas, y señalado por superiores de todos a los PP. Cesáreo González y Miguel Cabeza; y por haber tenido que salir de Buenos Aires también sin pasaporte el P. González, quedó al fin por único superior el P. Cabeza. Tentó Rosas el reconstruir la comunidad y hacerles abrir de nuevo el colegio del modo que él deseaba, valiéndose de diligencias del ministro Arana y de don Tomás Anchorena. Como paso preliminar, logró que se aceptase el partido de abrir por decreto del Gobierno el templo cerrado de San Ignacio y señalar como directores de él a los PP. Cabeza y Majesté. Mas cuando después se procuró juntar los demás para el colegio, siempre les halló resistentes: y en esto y en otras cosas conoció que ni aun salidos el P. Parés y el P. Berdugo los podía gobernar, porque tenían algún superior inmediato y obedecían al superior mayor que estaba en Río de Janeiro y luego en Montevideo; cosas todas que el Dictador no podía soportar. Poco a poco, iban obteniendo pasaportes varios y, salidos de Buenos Aires, recibían diversos destinos del P. Superior. Dos de los que en Buenos Aires quedaban recibieron por causas justificadas sus dimisorias: eran los PP. Ildefonso García y Francisco Majesté". (Hernández, 1914, p. 23)

Comparando estas líneas con la Historia secreta, que, conviene recordarlo, es conocida por Pérez y, probablemente, por Hernández, se observan llamativos silencios. Hernández calla que la distribución de sujetos respondía a la desconfianza que Berdugo sentía por varios de ellos; que nombró superiores a los padres González y Cabeza porque se llevaban muy mal entre ellos; que González se ve obligado a huir de Buenos Aires por las consecuencias de una inversión económica que hizo contra las ordenes de Berdugo y que la reapertura del templo generó un grave enfrentamiento entre los jesuitas. Es más, escribir que García y Majesté "recibieron por causas justificadas sus dimisorias" era una forma suave de resumir el conflicto. 


\section{La relectura de la historia como instrumento de construcción de la identidad: los jesuitas y Rosas}

Isérn -autor plenamente arraigado en Argentina- es, sin duda, el más comprensivo con Rosas. Recordando las obras de Mansilla (1898) y Alameda (1935) insiste en que, si cierta historia considera a Rosas símbolo de tiranía, la imparcial está dispuesta a hacer reservas de consideración. Subió al poder "destinado por la Providencia" e inspirando grandes esperanzas, con el apoyo de los mejores y, ya fuese por convicción o por cálculo político, se preocupó de poner fin al estado lamentable en que se hallaba la sociedad y la iglesia por culpa de Rivadavia. Está, incluso, dispuesto a explicar o justificar la violencia de su segundo mandato, preguntándose si fue solo él el responsable de toda la que se cometió. Dicho esto, el conflicto con los jesuitas se narra con brevedad e, incluso, distancia. Cuando Rosas se enfrenta a crecientes dificultades, causadas por sus enemigos y su política absorbente, exacerbado su carácter, responde con violencia. Los jesuitas, por su parte, quisieron servir a todos, convencidos de que había personas de buena voluntad tanto entre los federales como entre los unitarios. El resultado inevitable fue decretar la expulsión de los jesuitas que no se secularizasen. Sólo dos lo hicieron: Majesté y García, que "desde años atrás se habían acomodado a las exigencias del Dictador, revelándose contra su legítimo superior" y tuvieron que ser despedidos después de intentar todo lo posible para "conservarles en su vocación" (Isérn, 1936, p. 130). No es menos significativo y en este sentido está apuntando las posiciones, posteriores de Gracia y, sobre todo, Furlong, que haya eliminado cualquier mención - presente en los autores anteriores- a que la condición de extranjeros de los jesuitas les resulto ventajosa para el éxito de su misión y haya reducido, prácticamente hasta hacerla desaparecer, cualquier tensión entre la Compañía y el clero local.

Joaquín Gracia aun reconociendo que Rosas es un hombre muy discutido en la Historia y que hasta su llegada no se puso fin a años de anarquía, recupera la imagen anterior. Rosas, un político despótico, queriendo, por un lado, imponer a los jesuitas la propaganda de su política y los jesuitas, por otro, manteniéndose, fieles a su instituto, ajenos a la política. Como resultado, a los jesuitas, unidos estrechamente entre sí y bajo su legítimo superior, no les quedó otra salida que marcharse de Buenos Aires para evitar mayores desgracias. En esta visión, los jesuitas secularizados son prescindibles y se les cita brevemente y de pasada.

Consciente de quienes son sus lectores, Gracia amplia el protagonismo de las provincias, que reclaman, una y otra vez, la llegada de jesuitas, pero Rosas, teniéndolos casi prisioneros en Buenos Aires, lo dificulta. Aun así, Berdugo encuentra en las provincias - especialmente en Córdoba- un refugio frente a la arbitrariedad y las amenazas rosistas. Mientras la crítica al clero argentino, tan presente en Cotanilla, Pérez y Hernández, ha quedado reducida a la complicidad en la conspiración antijesuita de un eclesiástico —el canónigo Palacios-, al que ni siquiera cita por su nombre.

Las primeras revisiones de la figura de Rosas no parece que ejercieran influencia alguna en los textos jesuitas, pero no se puede decir lo mismo del revisionismo desarrollado después de la Primera Guerra Mundial y protagonizado por Carlos 


\section{Francisco Javier Gómez Díez}

Ibarguren, Julio Irazusta o Manuel Gálvez; un revisionismo que no es ajeno a una deriva nacionalista y autoritaria. Como reacción a este nacionalismo pesimista y dentro de un esfuerzo de consolidación historiográfica, se desarrollan los esfuerzos de Ricardo Levene al frente de la Academia Nacional de la Historia, donde se inserta activamente Guillermo Furlong.

Es necesario distinguir entre lo que escribe Furlong en el capítulo 25, "Los jesuitas y el dictador Rosas", de su obra Los jesuitas y la cultura rioplatense (1933) y lo que expone años después (Furlong, 1944). En 1933 se muestra muy moderado en su crítica a Rosas. Cuando el colegio prosperaba, escribe, los jesuitas "como sacerdotes de la iglesia de Dios y como educadores conscientes de su misión no quisieron abanderarse en ninguno de los dos partidos que cruel guerra se hacían a la sazón: unitarios y federales" y, así, antes que ceder a las exigencias arbitrarias de Rosas prefirieron alejarse del país. Da la impresión de que se marchan voluntariamente, y no expulsados. "Por desgracia, dos de [ellos] cometieron el error, bien perdonable a la sazón, de alabar en alguna forma la conducta del dictador". Fueron expulsados de la Compañía, manifestándose con ocasión de esto la prudencia de los superiores y el espíritu nada apostólico de Majesté.

Cuando, años después, vuelve a escribir sobre el mismo tema su planteamiento ha variado significativamente. Pretendiendo exponer con objetividad lo que se desprende de los documentos, dice no contarse ni entre los admiradores ni entre los detractores de Rosas. Aun así, le acusa de caprichoso, extravagante, mezquino, soberbio y dictador, de tener muy escasos principios y de haber perjudicado más que nadie a la educación en Argentina. Difícilmente podría ser más crítico, pero comienza con una disculpa histórica: Rosas no fue más que la "lógica consecuencia del delirium tremens de don Bernardino Rivadavia", a éste hay que responsabilizar de los hechos aciagos sucedidos entre 1835 y 1852.

Ya sea por la época en la que redacta su obra o por su temática, desarrolla un planteamiento novedoso: los jesuitas, educadores en ciudadanía, fueron una amenaza para Rosas. El progreso del colegio ofrecía a la República "jóvenes capaces ya de discurrir sobre la base de sólidas y sanas doctrinas, y por lo mismo ya no podrían admitir a ojos cerrados que el único bien social estaba en la santa federación" (Furlong 1944, p. 431); es decir, cuantos mayores fueran los frutos de moralidad y ciencia del colegio, mayores serían los obstáculos contra la mala política. En su obra anterior había apuntado esta idea, insistiendo en la valía de todos aquellos hombres educados en el colegio jesuita: "Rawson, Segui, Navarro Viola, Gorostiaga y tantísimos otros de aquella generación", junto a los llamados a ser los más destacados hombres de iglesia de las décadas posteriores.

Pese a presentar la conspiración final del Felipe Palacios, es muy generosa la imagen que ofrece del clero local, con encendidos elogios a diversos religiosos y reconociendo lo bien que fueron recibidos los jesuitas por la autoridad eclesiástica. Con todo, lo realmente interesante es la discreta presentación de Majesté y García y la imagen de unidad de la comunidad jesuita en torno, como siempre, a Berdugo, un hombre excepcional que deseaba dejar el cargo de superior para irse a misionar 


\section{La relectura de la historia como instrumento de construcción de la identidad: los jesuitas y Rosas}

indígenas. En este sentido es evidente que Furlong está jugando con un esquema muy repetido: la obediencia como virtud del súbdito acompañada del gobierno como servicio del superior (cfr. "Avisos para los que van por primera vez a las Anrillas”, La Habana 1877; AESI-A, estante 2, caja 75)

García casi no aparece, Majesté es varias veces elogiado y su salida no se vincula, como en autores anteriores, a explícitas conspiraciones ni da lugar a reflexiones tremendistas sobre su traición. Aunque apoyar a Rosas ya no le parece, como en su obra anterior, "un error bien perdonable" sino, como habría dicho Berdugo, la conducta equivocada de un sujeto dado a condescender y complacer a los hombres aun a costa de intereses más sagrados. Furlong reconoce en Majesté egregias cualidades que le hicieron muy popular. Sin ser hombre de talento, era de fácil y galana palabra, erudito, de conversación amena y, sobre todo, brillante en el púlpito "por la variedad de la argumentación, claridad de conceptos, melosidad de la voz, propiedad y elegancia de los gestos". Cita, como había hecho Cotanilla, una carta de Berdugo en la que, el 30 de enero de 1851, escribe lo que sabe de los exjesuitas. La carta no es la misma. Cotanilla ha buscado noticias terribles sobre las desgracias de los que perdieron su vocación. Furlong se limita a proporcionar noticias, unas mejores y otras peores, sobre la vida secularizada de estos hombres.

Por último - ya no sorprende-, Furlong ha anulado cualquier imagen de ruptura o peligro para la comunidad. Afirmando que la mayoría de los jesuitas eran cultos, virtuosos, rectos y equilibrados, sólo lamenta que algunos fueran menos serenos y valientes que su superior, aun pareciéndole comprensible, pues "temían no sin razón de la falange de gauchos asesinos lo que del populacho de Madrid en el año de 1834, de lo cual todos o casi todos habían sido testigos" (Furlong 1944, p. 466). Este esquema y, sobre todo, la referencia explícita a los gauchos, en relación con el papel - ya señalado - de los jesuitas como forjadores de la ciudadanía, tiene una importancia capital para la imagen construida por Furlong que hace de la Compañía un sujeto activo, y decisivo, en la configuración de la nación argentina.

La imagen del conflicto es especialmente significativa si consideramos el tiempo transcurrido desde la expulsión y la documentación que Furlong dice consultar. En su largo estudio sobre el Colegio de El Salvador, sigue muy de cerca lo que él llama Diario del padre Berdugo y la obra de Rafael Pérez; casi como todos los anteriores va cosiendo documentos y parafraseando, cuando no citando textualmente, a sus predecesores - con una tan explícita como irreal pretensión de objetividad- y, sobre esta base, amplia o concreta algunas cuestiones (obras para adaptar el local del colegio; datos de colegiales, estudios, etc.). Sin citar prácticamente a ningún autor no jesuita —una de las pocas excepciones es la reciente obra de Gálvez (1940)—, se apoya en los padres Leturia, Pérez y Berdugo, pero ¿en qué “texto" de Berdugo? El Diario, si existe yo, hoy por hoy, no lo he encontrado. Furlong afirma, en su Historia del Colegio del Salvador, haber publicado en el volumen LV (1936) de la revista bonaerense Estudios el Diario íntegro del P. Berdugo, pero, en dicho volumen, sólo existe un artículo firmado por Furlong, "Entrevista del Padre Berdugo con don Pedro de Angelis", que no es más que un fragmento - breve, aunque 


\section{Francisco Javier Gómez Díez}

significativo- de la Historia Secreta. El archivo jesuita de Buenos Aires podría aclarar muchas de estas dudas, pero, lamentablemente, permanece cerrado.

\section{Conclusiones}

La Compañía construye una identidad, la difunde entre los suyos y la convierte en el fundamento de un discurso apologético. Lo hace recurriendo a la relectura de la historia. Al tiempo, pone en marcha un esfuerzo, quizás no del todo consciente, por superar la ruptura con la modernidad; por insertarse en una sociedad que había rechazado en las primeras décadas del siglo XIX. Esa identidad está marcada por cuatro rasgos:

- Se presenta como una institución apolítica y educadora. No cabe ignorar ni la imagen negativa de lo político, ni la insistencia, como nunca lo había hecho antes, en sus tareas educativas, pese a la idealización de la experiencia de las misiones paraguayas.

- $\quad$ Construye, desde la narración permanente de su condición de perseguida, una imagen martirial $\mathrm{y}$, al mismo tiempo, la de una comunidad especialmente elegida por Dios.

- Una estructura interna sólidamente integrada, donde prima, como señalé más arriba, el esquema obediencia/servicio, forzando a silenciar sus tensiones internas.

- Una estrecha vinculación al papado - tanto un rasgo de identidad como instrumento de protección-, que si por un lado se presenta como razón de su éxito, frente al desorden de otras órdenes (así lo presenta, por ejemplo, Cotanilla en la entrada de su Diario del 6 de noviembre de 1858; AESI-A, C-95), por otro, plantea el problema de la supresión de 1773 que, durante todo el periodo, es silenciada, afirmando de forma explícita la continuidad de la antigua y la nueva Compañía.

Con respecto al análisis político, mientras se suaviza el tono agresivo inicial, de Cotanilla y, en menor medida, Pérez y Hernández, se abandona la imagen de la independencia americana como tragedia, para dar paso a la indiferente aceptación y, por fin, a convertir, en la obra de Furlong, a los jesuitas en legitimadores y promotores de esa independencia y en constructores de la nacionalidad. Con los autores y el lugar de publicación de las obras cambia el ámbito de interés de lo internacional a lo nacional. Con independencia de la nacionalidad de los diversos autores se desarrolla un progresivo enraizamiento de éstos en Argentina. Puede hablarse de la sustitución de historiadores españoles (Cotanilla, Pérez y Hernández) por otros argentinos o argentinizados (Isern, Gracia y Furlong) que, paralelamente, van reduciendo la tensión con el clero nacional. En el proceder historiográfico de Furlong se manifiesta claramente el proceso de confluencia entre la iglesia y el estado argentino y, por supuesto, la construcción del mito de la nación católica.

Este proceso se vio favorecido por la paradójica originalidad rosista, que, prolongando la política de Rivadavia, puede, a un tiempo, defender una tradición católica y expulsar a los jesuitas, porque su antijesuitismo, frente al de época 


\section{La relectura de la historia como instrumento de construcción de la identidad: los jesuitas y Rosas}

posteriores, es circunstancial y accesorio (Di Stefano 2010) y, al mismo tiempo, lo fue también por la construcción de una historiografía jesuita científica, pretendidamente objetiva e, incluso, académica, donde se insiste en la objetividad del documento, la colaboración entre investigadores y la apertura a un público lector cada vez más amplio.

\section{Bibliografía}

Alameda, J. (1935). Argentina católica. Buenos Aires: PP. Benedictinos.

Alcántara Bojorge, D. (2010). El proyecto historiográfico de Claudio Aquaviva y la construcción de la historia de la Compañía de Jesús en la Nueva España a principios del siglo XVII. Estudios de Historia Novohispama (40: 57-82).

Batllori, M. (1966). La cultura hispano-italiana de los jesuitas expulsos. Gredos: Madrid.

Betrán, J. L. (2010). El bonete y la pluma: la producción impresa de los autores jesuitas españoles durante los siglos XVI y XVII. En J. L. Betrán (ed.); La Compañía de Jesús y su proyección mediática en el mundo hispánico durante la Edad Moderna. Silex: Madrid.

Cárdenas Ayala, E. \& R. Di Stefano (2014). Los jesuitas en los orígenes de la Patria. En P.-A. Fabre \& E. Cárdenas \& J. H. Borja (coord.); La Compañía de Jesús en la América Latina después de la Restauración: los símbolos restaurados. Universidad Iberoamericana / Pontificia Universidad Javeriana: México / Bogotá.

Chinchila. P. (2014). Del Ars a la Monumenta: entre ciencia y amplificación. En P. Chinchilla \& A. Mendiola \& M. M. Morales; Del Ars Historica a la Monumenta Historica: la historia restaurada. Universidad Iberoamericana / Pontificia Universidad Javeriana: México / Bogotá.

Di Stefano, R. (2010), Ovejas negras. Historia de los anticlericales argentinos. Buenos Aires: Sudamericana.

Di Stefano, R. (2006), El laberinto religioso de Juan Manuel de Rosas, Anuario de Estudios Americanos (63:1).

Fernández Techera, J. (2007), Jesuitas, masones y Universidad. Montevideo: Ediciones de la Plaza.

Furlong, G. (1933). Los jesuitas y la cultura rioplatense. Montevideo: Urta y Curbelo. 


\section{Francisco Javier Gómez Díez}

Furlong, G. (1944). Historia del Colegio del Salvador y de sus irradiaciones culturales y espirituales en la ciudad de Buenos Aires, 1617-1943, vol. I. 1617-1841. Buenos Aires: Colegio de El Salvador.

Gálvez, M. (1940). Vida de don Juan Manuel de Rosas. Buenos Aires: El Ateneo.

Gómez Díez, F. J. (2016). Las reducciones del Paraguay en la Historia de la misión colombiana de la Compañía de Jesús, escrita por el P. José J. Cotanilla, S. J. Ponencia presentada en XVI Jornadas Internacionales sobre las misiones jesuíticas, Resistencia. Recuperado de: http://www.iighi-conicet.gob.ar/otras-publicaciones/

Gómez Díez, F. J. (2018). Expulsados que no disueltos. La interpretación de la supresión (1767-1773) en la obra del P. José J. Cotanilla, S.J. (1818-1886). En I. Fernández Arrillaga \& Mateo Ripoll \& Pacheco Albalate \& R. Tribaldos Soriano (eds.); Memoria de la expulsión de los jesuitas por Carlos III. Anaya: Madrid.

Gracia, J. (1940). Los jesuitas en Córdoba. Buenos Aires: Espasa Calpe.

Guasti, N. (2006). L'esilio italiano dei gesuiti spagnoli. Identità, controllo sociale e pratiche culturali (1767-1798). Roma: Edizioni di Storia e Letteratura.

Hernández, P. (1886). Juicio crítico sobre la educación antigua y la moderna. Buenos Aires: Pablo E. Coni e hijos.

Hernández, P. (1914). Reseña histórica de la misión de Chile-Paraguay de la Compañía de Jesús desde su origen en 1836 hasta el centenario de la restauración de la Compañía en 1914, Barcelona: Ibérica.

Imolesi, M. E. (2014). Dos historiadores modernos de las antiguas reducciones del Paraguay: Pablo Hernández y Guillermo Furlong. En P.-A. Fabre \& E. Cárdenas \& J. H. Borja (coord.); La Compañía de Jesús en América Latina después de la restauración: los símbolos restaurados. Universidad Iberoamericana / Pontificia Universidad Javeriana: México / Bogotá.

Isérn, J. (1936). La formación del clero secular de Buenos Aires y la Compañía de Jesús. Buenos Aires: San Miguel.

Lozier Almazán, B. (2018). Pedro de Angelis. Buenos Aires: Sammartino.

Mansilla, L. V. (1898). Rozas. Ensayo histórico-psicológico. París: Garnier.

Morales, M. M. (2014). La fábrica de la historia. En P. Chinchilla \& A. Mendiola \& M. M. Morales; Del Ars Historica a la Monumenta Historica: la historia restaurada. Universidad Iberoamericana / Pontificia Universidad Javeriana: México / Bogotá. 


\section{La relectura de la historia como instrumento de construcción de la identidad: los jesuitas y Rosas}

Palomo, F. (2005). Corregir letras para unir espíritus. Los jesuitas y las cartas edificantes en el Portugal del siglo XVI. Cuadernos de Historia Moderna (IV, 57-81).

Pérez, R. (1901). La Compañía de Jesús restaurada en República Argentina y Chile, el Uruguay y el Brasil. Barcelona: Henrich y C. .

Telesca, I. \& Perrone, N. (2014). El regreso frustrado de los jesuitas al Paraguay. En M. T. Matabuena, M. E. Ponce Alcocer \& J. E. Salcedo Martínez (coord.); La restauración de la Compañía de Jesús en la América hispanolusa. Universidad Iberoamericana / Pontificia Universidad Javeriana: México / Bogotá.

Recibido: 25/12/2019

Evaluado: 01/02/2020

Versión Final: 15/02/2020 